\title{
MRI contrast agents and nephrogenic systemic fibrosis: why physicians should be concerned
}

\author{
P. K. Chhetri
}

Assistant Professor, Department of Radiodiagnosis, College of Medical Sciences, Bharatpur, Chitwan district, Nepal

\begin{abstract}
Magnetic Resonance Imaging (MRI) as a diagnostic tool is becoming increasingly available in Nepal and more and more patients undergo MRI examination today. It was also traditionally believed that MRI contrast agents are safer than Computed Tomography (CT) contrast agents and MRI was indicated where CT contrast agents were contraindicated. Of special note must be made of a rare and newly recognized disease that was described in patients with renal disease and given MRI contrast agents. It is thus of increasing importance for the radiologists and the physicians alike to be aware of this rare and newly recognized disease-nephrogenic systemic fibrosis.
\end{abstract}

Key words: MRI contrast, computed tomography, nephrogenic systemic fibrosis.

\section{Introduction}

It is striking that many radiologists are still unaware that Nephrogenic Systemic Fibrosis (NSF) may be a serious late adverse reaction to gadolinium $(\mathrm{Gd})$ based contrast media used in Magnetic Resonance Imaging (MRI) despite the fact that the Food and Drug Administration (FDA) issued a warning on 8 June $2006 .{ }^{1}$ Furthermore, the warning from the vendor of gadodiamide (Omniscan ${ }^{\circledR}$, GE Health Diagnostic, Amersham, United Kingdom) issued on 6June 2006 has not been distributed to several countries. ${ }^{2}$

NSF is a rare disease that has been described with increasing frequency in the medical literature. This entity was first recognized in several patients in 1997 and was first described in the literature in $2000 .{ }^{3}$ Approximately 335 cases have been reported to date to the International

Correspondences: Dr. P. K. Chhetri

E-mail:pramodchhetri@rediffmail.com
Center forNephrogenic Fibrosing Dermopathy Research (ICNFDR). ${ }^{4}$ Originally, it was termed Nephrogenic Fibrosing Dermopathy (NFD) because this fibrosing skin condition occurred exclusively in patients with renal failure.

Specific histologic findings are associated with this condition including thickened collagen bundles with surrounding clefts, mucin deposition, and increased numbers of fibrocytes and elastic fibers. Later autopsy series of this disease have shown systemic manifestations including fibrosis of the skeletalmuscle, bone, lungs, pleura, pericardium, myocardium, kidney, muscle, bone, testes, and dura. ${ }^{5,6}$ Thus, the terminology recently has changed from NFD to NSF to reflect this systemic involvement. This condition can be quite disabling because the skin tighteningandmusculotendinous involvementresultinjoint contractures that can reduce the range of motion of joints. Some patients become severely disabled due to contractures, muscle weakness, and arthralgia. 
InAugust 2006, all members of the European Society of Urogenital Radiology (ESUR) received an electronic mail asking them to report cases of NSF to the chairman of the ESUR contrast media safety committee in request for material for the upcoming meeting of the committee. ${ }^{7}$ Three striking responses were obtained: (1) I have never heard about NSF (most frequent answer); (2) we have never seen a case of NSF; (3) yes, we have seen cases of NSF after administration of Gd-based contrast media. If the answer belonged to group 2 or 3 , the responder was asked which contrast agent they had used. In Europe, it turned out that more than 150 patients had developed NSF after exposure to a Gd-based contrast medium. The overwhelming majority ( $90 \%$ ) had gadodiamide. However there were no reports of NSF in patients with normal kidney function. Around 200 million patients have had injections of a gadolinium-based contrast agent since the early 1980s. A population of more than 30 million patients has received gadodiamide. So, in patients without end stage renal disease, all gadolinium-based contrast agents seem to be safe.

Grobner was the first to propose that MRI contrast media containing $\mathrm{Gd}$ might be a trigger of NSF. ${ }^{8} \mathrm{MRI}$ and Magnetic Resonance Angiography (MRA) examinations are commonly requested forpatients with endstage liver and renal disease to evaluate fortransplant eligibility, visualization of vascular anatomy, and posttransplant complications. Gadolinium-enhanced MRI traditionally has been preferred over contrast-enhanced computed tomography (CT) because many of these patients have impaired renal function. Gadolinium-based contrast media (at doses of $0.1-0.2 \mathrm{mmol} / \mathrm{kg}$ ) are consideredless nephrotoxic than iodinated contrast agents used in CT.${ }^{9}$ Despite numerous studies on the safety of gadolinium-based contrastagents in renal insufficiencyand dialysis patients, none had reported NSF as a complication before Grobner's study. ${ }^{8}$

\section{Pathogenesis}

Marckmann et al ${ }^{10}$ have postulated that NSF may result froma toxic reaction from free gadolinium $\left(\mathrm{Gd}^{3+}\right)$, liberated from the chelate but not adequately excreted due toimpaired renalfunction. Transmetallation, the release of free gadolinium from the chelate and subsequent binding to endogenous ions, is dependent on the molecular conditional thermodynamic stability. Gadoliniumcontrast media with lowerconditional stability constant valueswould be more likely toundergotransmetallation. The conditional stability constants (at a pH of 7.4) of the FDA approved major contrast agents are: gadodiamide, 10 $0^{14.9}$; gadoversetamide, 10 15.0; gadoteridol, 10 17.1; gadopentetate dimeglumine, $10^{18.1}$; and gadobenate dimeglumine, $10^{18.4} \cdot{ }^{11,12}$ If this postulate is true, contrast media such as gadodiamide and gadoversetamide, which have 1,000-fold lower conditional stability constants than themore stable gadolinium chelates, would be more likely to release free gadolinium and result in NSF. To minimize the risk of release of free gadolinium, contrast manufacturers have added additional chelate to bind the free gadolinium. The excess chelate content (according to the latest U.S. package inserts) of FDA-approved gadolinium contrast agents are as follows: gadodiamide $(12 \mathrm{mg} / \mathrm{mL})$, gadoversetamide $(28.4 \mathrm{mg} / \mathrm{mL})$, gadoteridol $(0.23 \mathrm{mg} / \mathrm{mL})$, gadopentetate $(0.4 \mathrm{mg} / \mathrm{mL})$, and gadobenate $(0 \mathrm{mg} / \mathrm{mL})$. Although some have theorized that the excess chelate with gadodiamide may contribute to thehigher incidence of $\mathrm{NSF}^{10}$, more likely the excess chelatehas a protective effectin binding the free gadolinium.

The Food and Drug Administration stated that all gadolinium-containing chelates are potentially associated with nephrogenic systemic fibrosis; however, most reported cases are linked to gadodiamide (Omniscan) and gadopentetate dimeglumine (Magnevist). In a comparative study to define the risks associated with 
P. K. Chhetri, MRI contrast agents and nephrogenic systemic fibrosis: why physicians should be concerned

each gadolinium-containing chelate, Reilly ${ }^{\mathbf{1 3}}$ examined their risk in hemodialysis population and concluded that the risk for nephrogenic systemic fibrosis with gadoteridol (ProHance) in patients with renal failure on dialysismay be lower than with gadodiamide (Omniscan) and gadopentetate dimeglumine (Magnevist).

NSF has currently no treatment option and can be fatal. Prevention is thus vital. Based on recommendations from scientific literature and regulatory bodies, the following general steps should be taken into account when considering administration of a Gd-containing medium to patients at risk for developing NSF: ${ }^{14}$

- Evaluate the medical history before administration of Gd-containing contrast media

- Identify patients at risk

- Patients on dialysis

- Patients with severe renal impairment(including those with renal impairment in the context of hepato-renal syndrome or liver transplantation)

- Consider carefully the need for a contrast enhanced MR examination, taking into account the use of possible alternative imaging methods

- If a risk-benefit assessment indicates the need for MR imaging with Gd-containing contrast media, use the lowest dose of $\mathrm{Gd}$-containing contrast media that would provide the diagnostic information sought

- As a general medical consideration for hemodialysisdependent patients: Perform hemodialysis promptly after administration of Gd-containing contrast media

\section{Conclusion:}

In conclusion, NSF is a rare, newly described, disfiguring and potentially debilitating disease withouta consistently effective treatment or prevention regimen that appears to be strongly associated with or triggered by intravenous injection of Gd-based contrast media forMRI and MRA examinations in patients with acute orchronic renal insufficiency.

All physicians \& radiologists should be informed and be aware of this serious late adverse reaction. Cases of NSF with a positive history of exposure to gadoliniumbased contrast agents should be reported. The ESUR Contrast Media Safety Committee (http://www.esur.org) will appreciate receiving information. Adverse events to gadolinium contrast agents can also be reported at http:// www.fda.gov/medwatch/index.html.

\section{References}

1. Food and Drug Administration (2006) Public Health Advisory: gadolinium-containing contrast agents for magnetic resonance imaging (MRI): Omniscan, OptiMARK, Magnevist, ProHance, and MultiHance. Available at http://www.fda.gov/cder/drug/advisory/ gadolinium_agents.htm. Accessed 07 Sept 2006.

2. Flaten H (GE Healthcare) (2006) Dear Healthcare Professional. http://www.fda.gov/medwatch/safety/2006/ gadolinium_NFD-SF_dhcp.pdf. Accessed 07 Sept 2006.

3. Cowper SE, Robin HS, Steinberg HM, et al Scleromyxedema-like cutaneous disease in renal-dialysis patients. Lancet 2000; 356:1000 -1.

4. Cowper SE. Nephrogenic fibrosing dermopathy [NFD/ NSF] Web site, 2001-2006. Available at http:// www.icnfdr.org. Last accessed June 8, 2006.

5. Gibson SE, Farver CF, Prayson RA. Multiorgan involvement in nephrogenic fibrosing dermopathy; an autopsy case and review of the literature. Arch Path Lab Med 2006;130 : 209-12.

6. Daram SR, Cortese CM, Bastani B. Nephrogenic fibrosing dermopathy/nephrogenic systemic fibrosis: report of a new case with literature review. Am J Kidney Dis 2005;46 : $763-5$. 
7. Thomsen HS. Nephrogenic systemic fibrosis: a serious late adverse reaction to gadodiamide. Eur Radiol. 2006 December; 16(12): 2619-21.

8. Grobner T. Gadolinium-a specific trigger for the development of nephrogenic fibrosing dermopathy and nephrogenic systemic fibrosis? Nephrol Dial Transplant 2006;21:1104-8.

9. Prince MR, Arnoldus C, Frisoli JK. Nephrotoxicity of highdose gadolinium compared with iodinated contrast. J Magn Reson Imaging 1996; 6:162 -6.

10. Marckmann P, Skov L, Rossen K, et al. Nephrogenic systemic fibrosis: suspected causative role of gadodiamide used for contrast-enhanced magnetic resonance imaging. J Am Soc Nephrol 2006; 17:2359 -62.
11. Prince MR, Erel HE, Lent RW, et al. Gadodiamide administration causes spurious hypocalcemia. Radiology 2003; 227:639 -46.

12. Uggeri F, Aime S, Anelli PL, et al. Novel contrast agents for magnetic resonance imaging. Synthesis and characterisation of the ligand BOPTA and its Ln(III) complexes. Inorg Chem1995; 34:633 -42.

13. Reilly RF. Risk for Nephrogenic Systemic Fibrosis with Gadoteridol (ProHance) in Patients Who Are on LongTerm Hemodialysis. Clin J Am Soc Nephrol 2008;3: 74751.

14. Nephrogenic Systemic Fibrosis and Gadolinium containing MRI contrast media- a summary of current knowledge. Bayer Schering Pharma. Research and Development for Life. Version July 2007. 11 